\title{
Baja recurrencia del edema macular diabético focal, 6 meses después de la fotocoagulación
}

\author{
Low recurrence of diabetic macular edema, 6 months after focal photocoagulation
}

Virgilio Lima-Gómez* y Selma A. Somilleda-Ventura

Servicio de Oftalmología, Hospital Juárez de México, Ciudad de México, México

\section{Resumen}

Objetivo: Identificar la proporción de ojos que presentan recurrencia del edema macular diabético a los 6 meses de la fotocoagulación focal. Método: Estudio observacional, analítico, transversal y prospectivo, en pacientes con edema macular diabético tratados con fotocoagulación focal. Se identificaron la proporción y los intervalos de confianza del 95\% (IC 95\%) en los ojos que presentaron recurrencia, y se compararon las medianas de las variables anatómicas y la agudeza visual mediante U de Mann-Whitney. Resultados: Se evaluaron 145 ojos y solo 10 (IC 95\%: 2.8-11) presentaron recurrencia del edema macular diabético. Los promedios de grosor del punto central y del campo central fueron mayores en los ojos con recurrencia, pero la diferencia de promedio de cambio entre grupos no fue significativa. Conclusión: La proporción de recurrencia del edema macular 6 meses después de la fotocoagulación focal es menor que la reportada para otros tratamientos de esta enfermedad y menor también que en otros estudios en los que se utilizó fotocoagulación.

PALABRAS CLAVE: Edema focal. Edema macular diabético. Fotocoagulación focal. Recurrencia. Retinopatía diabética.

\begin{abstract}
Objective: To identify the proportion of eyes with recurrence of diabetic macular edema, six months after focal photocoagulation. Method: Non-experimental, analytical, cross-sectional and prospective study in patients with diabetic macular edema treated with focal photocoagulation. The proportion and 95\% confidence intervals (95\% Cl) of eyes with recurrence of edema were identified; the distribution of anatomical variables and visual acuity was compared between eyes with and without recurrence, using Mann-Whitney's U. Results: 145 eyes were evaluated, and only 10 (95\% Cl: 2.8-11) had a recurrence. Center point thickness and center field thickness means were higher in eyes with recurrence than in eyes without it, but the difference of change mean betwwen groups was not statistically significant. Conclusion: The proportion of recurrent diabetic macular edema recurrence six months after successful focal photocoagulation was lower than the one reported for other treatments of diabetic macular edema, and by recent studies that used photocoagulation.
\end{abstract}

KEY WORDS: Diabetic macular edema. Diabetic retinopathy. Focal edema. Photocoagulation. Recurrence.

\author{
Correspondencia: \\ *Virgilio Lima-Gómez \\ Av. Instituto Politécnico Nacional, 5160 \\ Col. Magdalena de las Salinas, \\ Del. Gustavo A. Madero \\ C.P. 06770, Ciudad de México, México \\ E-mail: forscher7@gmail.com
}

Fecha de recepción: 03-05-2018

Fecha de aceptación: 11-07-2018

DOI: 10.24875/CIRU.18000426
Cir Cir. 2019;87:272-277

Contents available at PubMed www.cirugiaycirujanos.com 


\section{Introducción}

El edema macular diabético es la causa más común de pérdida visual en diabéticos con cualquier grado de retinopatía1. Existen dos tipos de filtración capilar, focal y difusa ${ }^{2}$, que requieren distintos tipos de tratamiento ${ }^{3}$. La American Academy of Ophthalmology reconoce dos tratamientos válidos para el edema macular: la fotocoagulación focal, que se reserva para los casos de filtración localizada, y los fármacos antiangiogénicos intravítreos, que se utilizan preferentemente para el edema difuso ${ }^{4}$.

La fotocoagulación se centra en tratar directamente el sitio de fuga capilar y es resolutiva en un solo evento ${ }^{5,6}$, por lo que puede beneficiar a una población mayor con una menor cantidad de recursos. En contraste, las inyecciones intravítreas tienen un efecto transitorio ${ }^{7} y$ en general se requieren hasta cinco aplicaciones posteriores al tratamiento inicial para mantener su eficacia ${ }^{8}$. Sin embargo, se desconoce si la recurrencia del edema macular es menor en los pacientes tratados con fotocoagulación focal.

Con la finalidad de ampliar la cobertura de atención, sería valioso contar con una intervención que pudiera realizarse en una sola visita, siempre y cuando la recurrencia del edema después de este tratamiento fuera baja. Se desarrolló un estudio para identificar la proporción de ojos con edema macular diabético tratados con fotocoagulación focal que tuvieran recurrencia del edema 6 meses después del tratamiento, y si alguna de las características anatómicas posteriores al tratamiento se asociaba con este desenlace.

\section{Método}

Se desarrolló un estudio observacional, analítico, transversal y prospectivo, en el que se evaluó a pacientes con edema macular clínicamente significativo (EMCS) que hubieran sido tratados con fotocoagulación focal. La población objetivo fueron los pacientes con EMCS de la Ciudad de México y su área metropolitana; la población accesible fueron los pacientes tratados en un hospital general entre el 26 de marzo de 2008 y el 30 de abril de 2014. El estudio fue autorizado por los comités de investigación y ética en investigación del lugar donde se desarrolló, y siguió los lineamientos de la Declaración de Helsinki.

Se incluyeron pacientes con diabetes tipo 2, de ambos sexos, con edad de 25 a 80 años, con cualquier grado de retinopatía diabética y edema macular clínicamente significativo, con filtración focal en la angiografía con fluoresceína y patrón espongiforme en la tomografía de coherencia óptica. Se excluyeron los pacientes con opacidad de medios que impidieran la adquisición de un mapa rápido macular de adecuada calidad, y los que tenían cualquier otra enfermedad que disminuyera la agudeza visual 0 incrementara el grosor retiniano. Se eliminaron los pacientes que no asistieron a alguna de las evaluaciones, así como los que desarrollaron opacidad de medios o cualquier otra enfermedad que redujera la visión o aumentara el grosor retiniano después de la fotocoagulación, y aquellos en quienes el edema macular no desapareció 3 semanas después.

En todos los pacientes se midió la agudeza visual mejor corregida bajo refracción subjetiva, y se evaluaron el segmento anterior ocular y el fondo de ojo. El edema macular se diagnosticó bajo midriasis farmacológica, usando una lente para biomicroscopía, y fue tratado con una sola sesión de fotocoagulación focal por un solo especialista en retina, de acuerdo con los criterios del estudio ETDRS (Early Treatment Diabetic Retinopathy Study) $)^{9}$.

En todos los ojos se obtuvo un mapa rápido macular de $6 \mathrm{~mm}$ con el siguiente procedimiento estandarizado: midriasis $\geq 6 \mathrm{~mm}$ (tropicamida $0.8 \%$ y fenilefrina $0.5 \%$ ), inclusión del equivalente esférico y el eje anteroposterior, identificación del nivel retiniano con una alarma acústica, y optimización del eje $\mathrm{Z}$ y la polarización. Todos los mapas se obtuvieron con flash, entre las 9:00 y las 11:00 a.m., con el equipo de tomografía de coherencia óptica Stratus OCT (Carl Zeiss, Meditec, Dublin, CA). Se evaluaron la agudeza visual y el fondo de ojo a las 3 semanas de la fotocoagulación y 6 meses después, y se obtuvo un mapa rápido macular a las 3 semanas.

La variable dependiente fue la recurrencia del EMCS, definida como la presencia de engrosamiento en el ojo tratado a los 6 meses de la fotocoagulación focal. Las variables predictoras fueron la agudeza visual mejor corregida medida en equivalente decimal, el grosor del punto central (GPC), el grosor del campo central (GCC) y el volumen macular, antes de la fotocoagulación y 3 semanas después de ella.

Se identificaron la proporción y los intervalos de confianza del 95\% (IC 95\%) de los ojos que presentaron recurrencia. Las medianas de las variables anatómicas y la agudeza visual antes del tratamiento y 3 semanas después se compararon entre los ojos con y $\sin$ recurrencia mediante la prueba $U$ de Mann 
Tabla 1. Comparación de los promedios de agudeza visual y de las variables anatómicas antes y después del tratamiento en la muestra $(n=145)$

\begin{tabular}{lccc}
\hline Variable & Antes $^{*}$ & Después $^{*}$ & $\mathbf{p}^{\dagger}$ \\
\hline Agudeza visual & $0.55 \pm 0.27$ & $0.62 \pm 0.28$ & $<0.001$ \\
GPC & $207.9 \pm 27.3$ & $208.5 \pm 25.7$ & 0.67 \\
GCC & $175.1 \pm 30.4$ & $175.7 \pm 30.4$ & 0.77 \\
Volumen macular & $7.73 \pm 0.71$ & $7.62 \pm 0.66$ & 0.002
\end{tabular}

*Promedio \pm desviación estándar.

tPrueba t de Student para muestras relacionadas.

GCC: grosor del campo central; GPC: grosor del punto central.

Tabla 2. Comparación del promedio de las variables entre ojos con y sin recurrencia

\begin{tabular}{lccc}
\hline Variable & \multicolumn{2}{c}{ Recurrencia* $^{*} \mathbf{p}^{\dagger}$} \\
\cline { 2 - 3 } & Sí $(\mathbf{n}=\mathbf{1 0})$ & $\mathbf{N o}(\mathbf{n}=\mathbf{1 3 5})$ & \\
\hline Agudeza visual inicial & $0.5 \pm 0.3$ & $0.6 \pm 0.3$ & 0.26 \\
GCC inicial & $229.2 \pm 25.1$ & $206.3 \pm 26.8$ & 0.01 \\
GPC inicial & $202.6 \pm 26.4$ & $173 \pm 29.7$ & 0.002 \\
Volumen macular inicial & $7.95 \pm 0.6$ & $7.72 \pm 0.7$ & 0.33 \\
Agudeza visual a las 3 semanas & $0.4 \pm 0.3$ & $0.6 \pm 0.3$ & 0.05 \\
GCC a las 3 semanas & $231.8 \pm 21.7$ & $206.8 \pm 25.2$ & 0.005 \\
GPC a las 3 semanas & $210.8 \pm 37.7$ & $173.1 \pm 28.3$ & 0.002 \\
Volumen macular a las 3 semanas & $7.92 \pm 0.3$ & $7.60 \pm 0.7$ & 0.07 \\
\hline $\begin{array}{l}\text { *Promediotdesviación estándar. } \\
\text { tPrueba U de Mann-Whitney. } \\
\text { GCC: grosor del campo central; GPC: grosor del punto central. }\end{array}$ & & \\
\hline
\end{tabular}

Whitney. Se consideró significativo un valor de $p<0.05$. La información se almacenó y analizó con el programa SPSS IBM versión 19 para Windows.

\section{Resultados}

Se evaluaron 145 ojos de 100 pacientes con una edad de 26 a 78 años (promedio: 59.4; desviación estándar: \pm 8.3$)$. De ellos, 77 (53.1\%) ojos eran de pacientes del sexo femenino. La duración de la diabetes fue de 1 a 30 años (15 \pm 6.7$) ; 52$ (35.9\%) ojos pertenecían a pacientes tratados con insulina y $93(64.1 \%)$ a pacientes tratados con hipoglucemiantes orales o dieta. Además, 77 (53.1\%) ojos eran de pacientes con hipertensión arterial sistémica.

El grado de la retinopatía fue no proliferativa leve en 16 ojos (11\%), no proliferativa moderada en $81(55.9 \%)$ y no proliferativa grave en $10(6.9 \%) ; 38$ ojos tuvieron retinopatía proliferativa (26.2\%). El edema macular fue monofocal en 95 ojos (65.5\%).

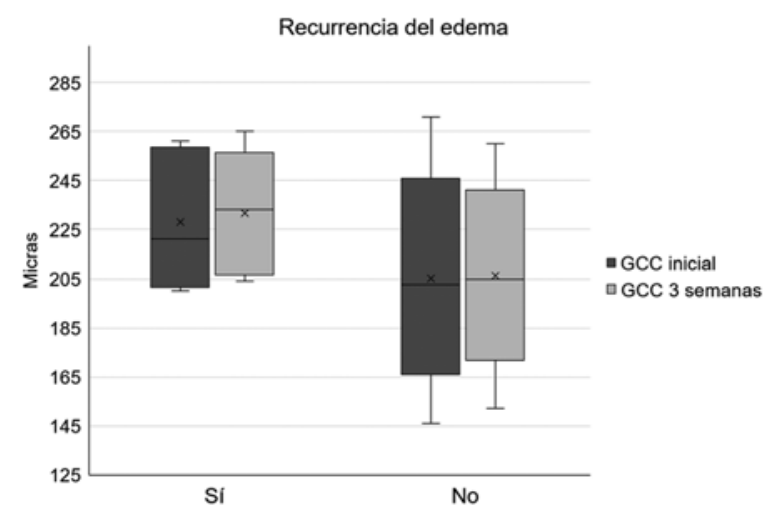

Figura 1. Comparación de la mediana y rango intercuartílico del grosor del campo central (GCC) entre grupos.

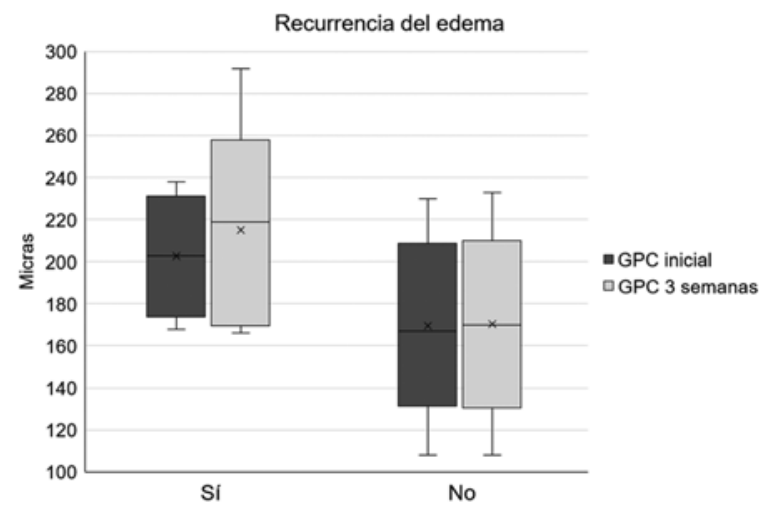

Figura 2. Comparación de la mediana y rango intercuartílico del grosor del punto central (GPC) entre grupos.

Antes de la fotocoagulación, la agudeza visual fue de 0.03 a $1.00(0.6 \pm 0.02)$, el GPC de 146 a $282 \mu \mathrm{m}$ $(207.9 \pm 2.3)$, el GCC de 107 a $264 \mu \mathrm{m}(175.8 \pm 2.5)$ y el volumen macular de 6.08 a $9.83 \mathrm{~mm}^{3}(7.7 \pm 0.06)$. A las 3 semanas de la fotocoagulación, la agudeza visual fue de 0.01 a 1.00 (0.6 \pm 0.02$)$, el GPC de 152 a $276(208.5 \pm 2.13)$, el GCC de 108 a $292(175.7 \pm$ 2.5) y el volumen macular de 6.03 a $9.53 \mathrm{~mm}^{3}(7.6 \pm$ 0.05). La agudeza visual aumentó y el volumen macular disminuyó estadísticamente en la muestra (Tabla 1).

Se observó recurrencia del EMCS en 10 ojos a los 6 meses de la fotocoagulación focal $(6.9 \%$; IC 95\%: 2.8-11). La comparación de las variables antes y después de la fotocoagulación (Tabla 2) identificó mayores promedios de GPC y GCC en los ojos que tuvieron recurrencia (Figs. 1 y 2). Mediante el análisis de sensibilidad no se identificó ningún punto de corte de estas variables que se asociaran significativamente con la recurrencia del engrosamiento. 
Tabla 3. Comparación del promedio de cambio de las variables entre ojos con y sin recurrencia

\begin{tabular}{|c|c|c|c|c|c|c|}
\hline \multirow[t]{2}{*}{ Variable } & \multicolumn{3}{|c|}{ Promedio de cambio absoluto* } & \multicolumn{3}{|c|}{ Promedio de cambio porcentual ${ }^{*}$} \\
\hline & Recurrencia & Sin recurrencia & $\mathbf{p}^{\dagger}$ & Recurrencia & Sin recurrencia & $\mathbf{p}^{\dagger}$ \\
\hline Agudeza visual & $-0.006 \pm 0.12$ & $0.07 \pm 0.2$ & 0.35 & $11.4 \pm 49$ & $26.6 \pm 80.3$ & 0.74 \\
\hline GPC & $8.2 \pm 21$ & $0.05 \pm 25.9$ & 0.89 & $3.8 \pm 9.3$ & $1.07 \pm 13.2$ & 0.44 \\
\hline GCC & $2.6 \pm 16$ & $-0.02 \pm 0.4$ & 0.26 & $1.5 \pm 6.9$ & $0.7 \pm 8.5$ & 0.93 \\
\hline Volumen macular & $-0.02 \pm 0.4$ & $-0.11 \pm 0.4$ & 0.36 & $-0.01 \pm 5$ & $-1.3 \pm 4.9$ & 0.24 \\
\hline
\end{tabular}

*Promedio \pm desviación estándar.

tPrueba U de Mann-Whitney.

GCC: grosor del campo central; GPC: grosor del punto central.

La comparación de los cambios absolutos de las variables identificó que solo los ojos sin recurrencia aumentaron el promedio de la agudeza visual; sin embargo, el volumen basal no difirió entre los ojos con y sin recurrencia, y la reducción del promedio de volumen macular en los últimos fue cinco veces el observado en los primeros. El promedio de cambio del GPC y del GCC después del tratamiento también fue mayor que en los ojos sin recurrencia (Tabla 3); las diferencias de promedio entre los grupos no fueron significativas debido a la baja proporción de pacientes que tuvieron recurrencia.

\section{Discusión}

En este estudio, la recurrencia del edema macular 6 meses después de la fotocoagulación focal fue menor del 10\%; en un escenario en que el tratamiento que recibe mayor difusión (terapia antiangiogénica) requiere múltiples procedimientos, la fotocoagulación focal resolvería el $93 \%$ de los casos con un solo evento.

El edema macular focal puede tratarse con inhibidores del factor de crecimiento vascular endotelial o con glucocorticoides por vía intravítrea, los cuales reducen el grosor retiniano y pueden mejorar la visión en poco tiempo ${ }^{10}$; sin embargo, ambas terapias tienen una alta tasa de recurrencia debido a su efecto transitorio, y pueden requerir hasta 12 procedimientos en 1 año para conseguir la resolución del edema ${ }^{11}$.

Wang, et al..$^{12}$ reportaron que los ojos tratados únicamente con una dosis de bevacizumab intravítreo $(n=21)$ o combinado con triamcinolona $(n=19)$ mostraron una proporción de recurrencia del 52.4 y el $47.4 \%$ respectivamente, a las 12 semanas. En un estudio en el que se comparó la eficacia de tres medicamentos antiangiogénicos en el edema macular diabético, inyectados cada 4 semanas durante $6 \mathrm{me}$ ses, el edema persistió en el 37\% (aflibercept), el 46\% (ranibizumab) y el $56 \%$ (bevacizumab) de los casos $^{13}$.

Liegl, et al. ${ }^{14}$, en un estudio prospectivo a 1 año, reportaron que el $84 \%$ de los pacientes con edema macular diabético tratados con ranibizumab requirieron inyecciones adicionales, en comparación con aquellos que recibieron terapia combinada con ranibizumab y fotocoagulación (35\%). Otro estudio en el que se usó bevacizumab combinado con fotocoagulación reportó que 13 de 23 ojos (57\%) con edema macular diabético no requirieron inyecciones adicionales después de 12 meses $^{15}$.

En los estudios Intravitreal Aflibercept injection in vision impairment due to diabetic macular edema (VIVID-DME) y Study of intravitreal Aflibercept injection in patients with diabetic macular edema (VISTA $\mathrm{DME})^{16}$, que evaluaron el aflibercept para tratar el edema macular diabético, el grupo de comparación al que solo se asignó fotocoagulación recibió tratamientos 12 semanas posteriores al primer procedimiento. Durante el seguimiento a las 48 semanas, esos ojos requirieron un promedio de 2.7 (VISTA) y 2.1 (VIVID) nuevos tratamientos de fotocoagulación. Si se asumiera una distribución normal, se estima que el $84 \%$ de los ojos tratados con fotocoagulación en el estudio VISTA y el $59 \%$ en el estudio VIVID tuvieron recurrencia.

Un ensayo clínico que evaluó 52 ojos tratados con fotocoagulación (focal y en parrilla) incluyó dos grupos de tratamiento adicional: bevacizumab solo o combinado con fotocoagulación; la agudeza visual mejoró y el grosor retiniano disminuyó en ambos, con una proporción de recurrencia del 20 y el $7.7 \%$ respectivamente, después de 5 meses $^{17}$.

En el estudio A 12 month core study to assess the efficacy and safety of Ranibizumab (intravitreal injections) in patients with visual impairment due to diabetic macular edema and a 24 month open-label extension study RESTORE ${ }^{18}$, que evaluó el ranibizumab para 
tratar el edema macular diabético, 52 de 110 ojos que recibieron solo fotocoagulación tenían edema focal (47.4\%); 70 de estos 110 ojos requirieron mas de un tratamiento, pero no se identificó cuáles tenían edema focal o difuso. Si todos los ojos que no requirieron retratamientos $(n=40)$ hubieran presentado edema focal, 12 de esos 52 ojos con tal característica habrían tenido recurrencia a los 6 meses (23.1\%), y la proporción podría ser mayor si algunos casos con edema difuso en el estudio hubieran requerido un solo tratamiento.

En comparación, una sola sesión de fotocoagulación focal interrumpe la filtración capilar y reduce el grosor retiniano ${ }^{19}$, es altamente costo-efectiva para reducir el riesgo de pérdida visual moderada ${ }^{20}$ y se considera el tratamiento estándar en los casos con agudeza visual $>20 / 30$ y con edema sin afección central ${ }^{21}$.

En este estudio, los promedios del GPC y del GCC antes de la fotocoagulación fueron menores en los ojos sin recurrencia que en los ojos con recurrencia; el número de los últimos fue reducido y no permitió identificar asociaciones con las variables anatómicas. Es necesario evaluar de manera prospectiva un mayor número de ojos con recurrencia para identificar predictores de este desenlace que podrían modificarse tempranamente.

Como la fotocoagulación no cambia el daño endotelial, la recurrencia del edema macular después del procedimiento no es una falla del tratamiento, sino un nuevo daño en la red capilar; identificar si el pronóstico visual empeora en los ojos con recurrencia requiere un análisis adicional en ensayos subsecuentes.

Los resultados de este estudio aplican para pacientes con EMCS focal; en el edema difuso, que generalmente tiene un engrosamiento y cierre capilar extenso ${ }^{21}$, los fármacos antiangiogénicos intravítreos han remplazado a la fotocoagulación. Además, el edema macular difuso se asocia con condiciones sistémicas como dislipidemia y nefropatía diabética, lo cual podría aumentar la proporción de ojos con edema recurrente ${ }^{22}$; estas variables tienen una menor contribución en el edema macular focal, en el que la filtración viene de uno o más sitios de daño localizados en la red capilar ${ }^{23}$.

Una fortaleza del estudio es que, en todos los ojos evaluados, se presentó una remisión del engrosamiento a las 3 semanas de la fotocoagulación, por lo que no se estudiaron ojos con edema persistente 0 refractario a la terapia. Una potencial debilidad es que no se contó con un grupo control, pero no se considera ético dejar pacientes sin tratar cuando existe un procedimiento terapéutico eficaz; sin embargo, metodológicamente hubiera sido conveniente comparar la proporción de recurrencia con un grupo que recibiera antiangiogénicos, pues por la duración del efecto de estos fármacos se hubiera esperado una recurrencia muy alta desde el primer mes posterior al procedimiento.

En una población diabética en la que el edema macular focal es más frecuente que el difuso, la fotocoagulación permitiría ampliar la cobertura de intervención para prevenir la pérdida visual moderada, debido a que es un tratamiento efectivo con baja proporción de recurrencia.

\section{Conclusión}

En resumen, la recurrencia del edema macular focal después de un tratamiento eficaz con fotocoagulación fue del $6.9 \%$. Se requiere identificar cómo cambia esa proporción en un periodo más largo y con un número mayor de sujetos para poder detectar las características asociadas con la recurrencia.

\section{Conflicto de intereses}

Los autores declaran que no tuvieron conflicto de intereses para desarrollar el trabajo ni para su publicación.

\section{Responsabilidades éticas}

Protección de personas y animales. Los autores declaran que para esta investigación no se han realizado experimentos en seres humanos ni en animales.

Confidencialidad de los datos. Los autores declaran que han seguido los protocolos de su centro de trabajo sobre la publicación de datos de pacientes.

Derecho a la privacidad y consentimiento informado. Los autores declaran que en este artículo no aparecen datos de pacientes.

\section{Bibliografía}

1. Rangasamy S, McGuire PG, Das A. Diabetic retinopathy and inflammation: novel therapeutic targets. Middle East Afr $\mathrm{J}$ Ophthalmol. 2013;19:52-9.

2. Zhang X, Zeng H, Bao S, Wang N, Gillies M. Diabetic macular edema: new concepts in patho-physiology and treatment. Cell Biosci. 2014;4:27.

3. Ford JA, Lois N, Royle P, Clar C, Shyangdan D, Waugh N. Current treatments in diabetic macular oedema: systematic review and meta-analysis. BMJ Open. 2013;3(3). pii: e002269.

4. Federman JL. Retina and vitreous text. St. Louis: Mosby-Year Book; 1994.

5. Techniques for scatter and local photocoagulation treatment of diabetic retinopathy: Early Treatment Diabetic Retinopathy Study Report no. 3. 
The Early Treatment Diabetic Retinopathy Study Research Group. Int Ophthalmol Clin. 1987;27:254-64.

6. Romero-Aroca P, Reyes-Torres J, Baget-Bernaldiz M, Blasco-Suñe C. Laser treatment for diabetic macular edema in the $21^{\text {st }}$ century. Curr Diabetes Rev. 2014;10:100-12.

7. Simó R, Carrasco E, García-Ramírez M, Hernández C. Angiogenic and antiangiogenic factors in proliferative diabetic retinopathy. Curr Diabetes Rev. 2006;2:71-98.

8. Dhibi HAA, Arevalo JF. Clinical trials on corticosteroids for diabetic macular edema. World J Diabetes. 2013;4:295-302.

9. Treatment techniques and clinical guidelines for photocoagulation of diabetic macular edema. Early Treatment Diabetic Retinopathy Study Report Number 2. Early Treatment Diabetic Retinopathy Study Research Group. Ophthalmology. 1987;94:761-74.

10. Lee HY, Lee SY, Park JS. Comparison of photocoagulation with combined intravitreal triamcinolone for diabetic macular edema. Korean J Ophthalmol. 2009;23:153-8.

11. Shimonagano Y, Doi N, Noda Y, Uemura A, Sakamoto T. Recurrence of diabetic macular edema after intravitreous injection of triamcinolone following vitrectomy. Jpn J Ophthalmol. 2007;5:278-84.

12. Wang YS, Li X, Wang HY, Zhang ZF, Li MH, Su XN. Intravitreal bevacizumab combined with/without triamcinolone acetonide in single injection for treatment of diabetic macular edema. Chin Med J (Engl). 2011;124: 352-8.

13. Wykoff CC, Hariprasad SM. Comparing aflibercept, bevacizumab, and ranibizumab for DME: analysis of DRCR Protocol T. Ophthalmic Surg Lasers Imaging Retina. 2015;46:302-5.

14. Liegl R, Langer J, Seidensticker F, Reznicek L, Haritoglou C, Ulbig MW, et al. Comparative evaluation of combined navigated laser photocoagulation and intravitreal ranibizumab in the treatment of diabetic macular edema. PLoS One. 2014;9:e113981.
15. Barteselli G, Kozak I, El-Emam S, Chhablani J, Cortes MA, Freeman WR. 12-month results of the standardised combination therapy for diabetic macular oedema: intravitreal bevacizumab and navigated retinal photocoagulation. Br J Ophthalmol. 2014;98:1036-41.

16. Korobelnik JF, Do DV, Schmidt-Erfurth U, Boyer DS, Holz FG, Heier JS, et al. Intravitreal aflibercept for diabetic macular edema. Ophthalmology. 2014; $121: 2247-54$.

17. Takamura $Y$, Tomomatsu T, Matsumura T, Arimura S, Gozawa M, Takihara $Y$, et al. The effect of photocoagulation in ischemic areas to prevent recurrence of diabetic macular edema after intravitreal bevacizumab injection. Invest Ophthalmol Vis Sci. 2014;55:4741-6.

18. Mitchell P, Bandello F, Schmidt-Erfurth U, Lang GE, Massin P, Schlingemann RO, et al. The RESTORE study. Ranibizumab monotherpy or combined with laser versus laser monotherapy for diabetic macular edema. Ophthalmology. 2011;118:615-25.

19. Neubauer AS, Langer J, Liegl R, Haritoglou C, Wolf A, Kozak I, et al. Navigated macular laser decreases retreatment rate for diabetic macular edema: a comparison with conventional macular laser. Clin Ophthalmol. 2013;7:121-8.

20. Stein JD, Newman-Casey PA, Kim DD, Nwanyanwu KH, Johnson MW, Hutton DW. Cost-effectiveness of various interventions for newly diagnosed diabetic macular edema. Ophthalmology. 2013;120:1835-42.

21. Mitchell P, Wong TY. Management paradigms for diabetic macular edema. Am J Ophthalmol. 2014;157:505-13.

22. Koo NK, Jin HC, Kim YC. Relationship between the morphology of diabetic macular edema and renal dysfunction in diabetes. Korean J Ophthalmol. 2013;27:98-102.

23. Perente I, Alkin Z, Ozkaya A, Dardabounis D, Ogreden TA, Konstantinidis $A$, et al. Focal laser photocoagulation in non-center involved diabetic macular edema. Med Hypothesis Discov Innov Ophthalmol. 2014;3:9-16. 